\title{
Magnetic resonance imaging in Leber's optic neuropathy
}

\author{
A G KERMODE, I F MOSELEY, B E KENDALL, D H MILLER, D G MACMANUS, \\ W I MCDONALD
}

From the Multiple Sclerosis NMR Research Group, London, UK

SUMMARY Thirteen males with Leber's optic neuropathy had magnetic resonance imaging (MRI) of the brain, and in eight the optic nerves were imaged using STIR $^{1}$ (Short Time Inversion Recovery) sequences. All optic nerve scans were abnormal. In seven with bilateral visual loss four showed bilateral increased optic nerve signal and three unilateral increase. The involvement was of the mid and posterior intra-orbital sections over three $5 \mathrm{~mm}$ slices or more with sparing of the anterior portion. One patient with unilateral visual loss had increased signal only on the affected side. Brain MRI was normal, in marked contrast to the findings in clinically isolated optic neuritis in which multiple white matter lesions are seen in the majority.

Leber's optic neuropathy is an inherited disorder predominantly of young men and characterised by acute or sub-acute onset of central visual loss, usually painless. At the onset of visual loss the optic disc is swollen and has a marked increase in small vessels giving a "vasculitic" appearance ${ }^{23}$ (fig 1); there is however no leakage on fluorescein angiography. ${ }^{2}$ These ophthalmoscopic vascular changes have been noted to precede the visual loss and to be present in asymptomatic family members who are at risk. ${ }^{2}$ After the development of visual loss a capillary-poor retina with attenuated arterioles and a pale optic disc remain. $^{2-4}$ The visual loss is almost always bilateral, and in two thirds of cases both sides are affected simultaneously (the great majority within one month), although intervals of over one year have been recorded. ${ }^{56}$ The disease is inherited through the maternal line but does not follow Mendelian principles. Paternal transmission has never been reported. It is likely that cytoplasmic or mitochondrial transmission is involved. ${ }^{6-8}$

Additional neurological abnormalities including a multiple sclerosis-like syndrome have been described both clinically and in those coming to necropsy. ${ }^{9-12}$ Pathological studies are rare, as patients die of

Address for reprint requests: Professor W I McDonald, Institute of Neurology, Queen Square, London WCIN 3BG, UK.

Received 10 August 1988 and in revised form 28 November 1988. Accepted 12 December 1988 causes unrelated to their visual impairment. Atrophic changes in the retina with extensive optic nerve axonal degeneration with loss of myelin are always found.

In our experience in London the finding of clinical CNS abnormalities outside the optic nerves in Leber's disease is rare. MRI provides a sensitive means of detecting white matter abnormalities in the brain and we have therefore compared the frequency of CNS abnormalities as detected by MRI in Leber's optic neuropathy with clinically isolated acute optic neuritis in which silent lesions are common. ${ }^{13-17}$

\section{Methods}

Patients in whom a diagnosis of Leber's optic neuropathy had been made at Moorfields Eye Hospital, City Road or The National Hospitals, Queen Square and Maida Vale, London were recalled and the diagnosis reviewed. A characteristic family history, clinical presentation and ophthalmoscopic findings were required for inclusion in the study. Twelve of our thirteen patients were included in a previous genetic study. $^{18}$

Scanning was performed on a Picker 0.5 Tesla Magnetic resonance imager. In all cases spin echo $\left(\mathrm{SE}_{2000 / 60}\right)$ brain images were obtained with $245 \mathrm{~mm}$ contiguous sections. Eight patients (one with unilateral visual loss) had coronal imaging of the optic nerves using the STIR sequence ${ }^{1}\left(I_{1500 /}\right.$ ${ }_{40 / 150}$, six $5 \mathrm{~mm}$ contiguous slices) with a surface eye coil, and in six transverse STIR images were also obtained.

The images were examined independently by two neuroradiologists (IFM, BEK) and abnormalities accepted only if agreed by both. 


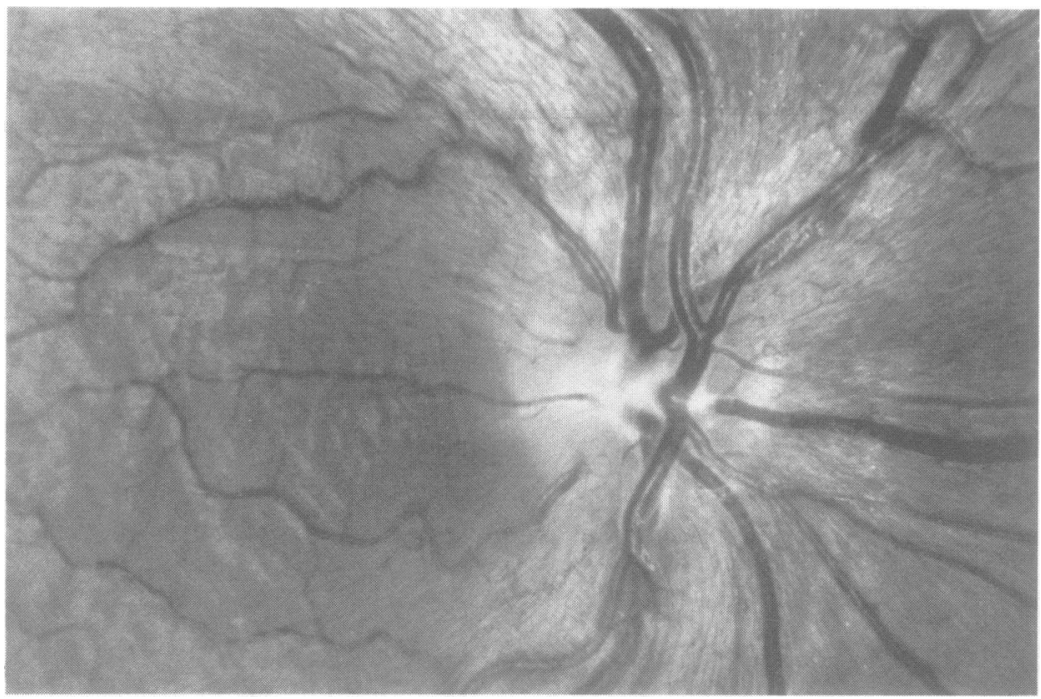

Fig 1 Fundal photograph (red free) of a patient in the acute stage illustrating optic disc swelling, a swollen nerve fibre layer and mild peri-papillary microangiopathy.

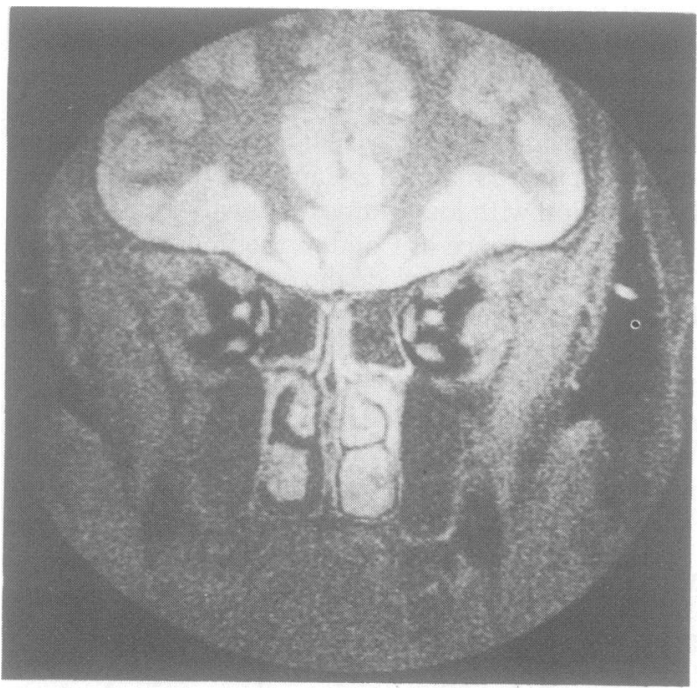

Fig 2 Coronal STIR image demonstrating bilaterally increased optic nerve signal.

\section{Results}

Clinical: Thirteen males were scanned, aged 19 to 44 years, mean 29. At the time of MRI twelve had bilateral visual loss of 8 months to 16 years duration, and one had unilateral visual loss of three months duration with bilateral ophthalmoscopic vascular changes. Of the 12 patients with bilateral visual loss, eight had bilateral simultaneous visual deterioration, and in all patients both eyes were severely affected within 7 months. Visual loss was severe in all patients, with visual acuities below $2 / 60$, though in three someo improvement occurred subsequently. Only one had an abnormal neurological sign (an isolated right extenso plantar response) other than those attributable to visual problems.

MRI: Abnormalities of the optic nerves were seen in alf eight patients in whom STIR images were obtained. In the seven with bilateral visual loss four had increased optic nerve signal bilaterally which extended over three contiguous $5 \mathrm{~mm}$ sections or more (figs 2,3 ). Two had unilateral increased signal over three or more slices; of these two, one had equivocally increased signal on the other side. The remaining patient with bilateral visual loss had an equivocal increase in signal unilaterally with normal appearance on the other side. In all patients the increase in signal was located in the mid to posterior intra-orbital portions of the nerves.

In no instance were the optic nerves unequivocally atrophic (although suspicious changes were present in one patient) nor was swelling seen, although only one patient was scanned in the acute stage. This patient was the only one with unilateral visual loss and there was unilateral high signal in the optic nerve corresponding to the affected side in the anterior to mid-orbital region over three contiguous $5 \mathrm{~mm}$ sections. There were "vasculitic" retinal changes on the unaffected side which were unaccompanied by MRI changes in the optic nerve. This patient has recently developed visual loss in the second eye, but it has not been possible for personal reasons to re-scan him.

The mean length of abnormal signal in the optic 


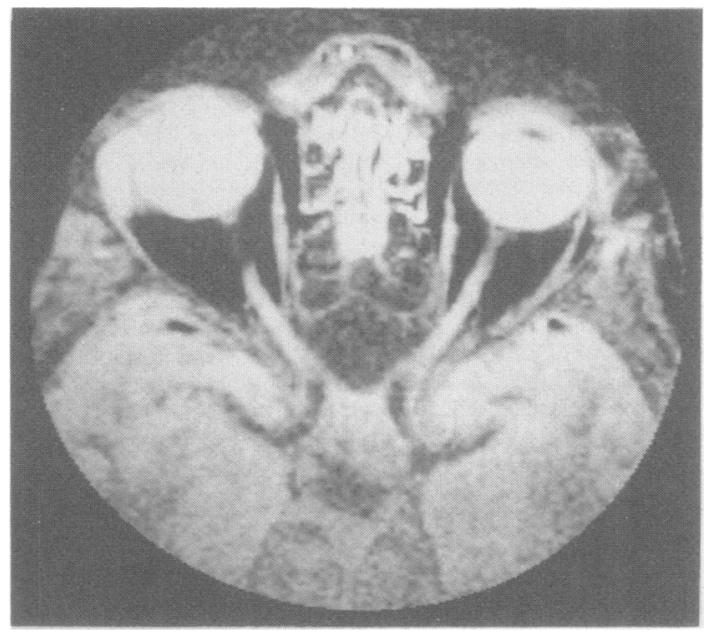

Fig 3 Transverse STIR image demonstrating high signal in the mid to posterior intra-orbital portion of both optic nerves.

nerve was 3.25 sections of $5 \mathrm{~mm}$, corresponding approximately to $15 \mathrm{~mm}$. The optic chiasm was poorly seen in the coronal STIR films due to the inherent problems of surface coil scaning in which the signal to noise ratio falls sharply at depth.

No patient had any abnormalities of the brain detected by MRI.

\section{Discussion}

This study has shown some important differences between the MRI changes in Leber's optic neuropathy and acute idiopathic clinically isolated optic neuritis. No MRI abnormalities were observed in the brain in Leber's optic neuropathy, whereas $50 \%$ to $68 \%$ of patients with clinically isolated unilateral optic neuritis have multifocal white matter lesions at presentation. ${ }^{13-17}$

The commonest abnormality in the optic nerves was bilaterally increased signal, usually extending over three $5 \mathrm{~mm}$ sections or more, and in all but one patient sparing the nerve immediately behind the eye. MRI in clinically isolated optic neuritis by contrast more frequently has anterior to mid-orbital high signal (45\% and $60 \%$ involved respectively) and a shorter longitudinal extent of lesions (mean 2.1 slices, or approximately $10 \mathrm{~mm}) \cdot{ }^{14} \mathrm{It}$ is of interest that the single patient who was scanned in the acute stage was the only one with involvement of the anterior portion of the optic nerve, raising the possibility that the anterior involvement reflects oedema which might resolve subsequently. Unfortunately it has not been possible to re-scan this patient. The total number of patients scanned is small, and serial scanning from the acute to the chronic phases of both Leber's optic neuropathy and idiopathic optic neuritis is required to establish whether the apparent differences regarding distribution of lesions are characteristic of each disease.

Unequivocal atrophy of the optic nerves was not seen, despite its reported frequency in necropsy studies. ${ }^{9112}$ Assessment of slight atrophy in optic nerve imaging is however difficult when there are bilateral abnormalities, and it could have been missed. In optic neuritis delayed and incomplete recovery correlates with the presence of longer MRI lesions, ${ }^{14}$ and the finding of a greater mean length of lesions in the patients with Leber's optic neuropathy is in keeping with this observation. In two patients however, there was severe bilateral visual loss (both with visual acuity less than $1 \cdot 5 / 60$ bilaterally) but only unilateral increased signal. Visual loss depends upon interference with axonal conduction, whereas MRI gives information principally about the fluid and cellular environment of the axons. The axonal environment of course influences conduction ${ }^{19}$ but the resolution of MRI at present is crude in comparison with the small changes which are adequate to disturb function. Discrepancies are therefore to be expected, as has been observed in MRI of optic neuritis. ${ }^{14}$

What is the origin of the abnormal signal in the optic nerves? The signal intensity in MRI depends upon many different factors including the density of protons and their immediate physico-chemical environment. In the acute stages the change in signal could be accounted for by oedema, ${ }^{20}$ though axonal swelling (for which there is ophthalmoscopic evidence in the peripapillary region of the retina ${ }^{3}$ ) may contribute. Gliosis is an important source of abnormal signal in chronic experimental lesions ${ }^{21}$ and from the pathology of the nerves in Leber's disease is likely to be a major component, particularly in the chronic phase.

Quantitative MRI may be able to differentiate between gliosis and oedema, ${ }^{2021}$ but the technique has not yet been successfully applied to the optic nerve because of its small size and the consequent partial volume effects, the high signal from orbital fat, data truncation and chemical shift artefacts, and movement of the eyes. Further technical developments should overcome these obstacles.

The Multiple Sclerosis NMR Research Group was established with a generous grant from the Multiple Sclerosis Society of Great Britain and Northern Ireland and the work is supported by them and the Medical Research Council. We are grateful to $\mathbf{M r}$ M Sanders for his advice and for providing the fundal photograph.

\section{References}

1 Johnson G, Miller DH, MacManus D, et al. STIR sequences in 
MRI imaging of the optic nerve. Neuroradiology 1987;29: 238-45.

2 Nikoskelainen E, Hoyt WF, Nummelin K. Ophthalmoscopic findings in Leber's hereditary optic neuropathy. Fundus findings in asymptomatic family members. Arch Ophthalmol 1982;100:1597-602.

3 Nikoskelainen E, Hoyt WF, Nummelin K. Ophthalmoscopic findings in Leber's hereditary optic neuropathy. The fundus findings in affected family members. Arch Ophthalmol 1983;101:1059-68.

4 Nikoskelainen E. The clinical findings in Leber's hereditary optic neuropathy. Leber's disease. Trans Ophthalmol Soc UK 1985;104(pt 8):845-52.

5 Wallace DC. A new manifestation of Leber's disease and a new explanation for the agency responsible for its unusual pattern of inheritance. Brain 1970;93:121-32.

6 Carroll WM, Mastaglia FL. Leber's optic neuropathy; a clinical and evoked potential study of affected and asymptomatic members of a six generation family. Brain 1979;102:559-80.

7 Nikoskelainen E, Sanontaus ML, Wayne OP, Katila MJ, Nummelin K. Leber's hereditary optic neuroretinopathy, a maternally inherited disease. Arch Ophthalmol 1987;105:665-71.

8 Nikoskelainen EK, Hassinen IE, Paljarvi L, Lang H, Kalimo H. Lebers hereditary neuroretinopathy, a mitochondrial disease? Lancet 1984;ii: 1474.

9 Went LN. Leber disease and variants. In: Vinken PJ, Bruyn GW, eds. Handbook of Clinical Neurology. Amsterdam: NorthHolland, 1971; Vol 13:94-110.

10 Lees F, Macdonald AME, Aldren Turner JW. Leber's disease with symptoms resembling disseminated sclerosis. $J$ Neurol Neurosurg Psychiatry 1964;13:415-21.

11 Adams JH, Blackwood W, Wilson J. Further clinical and patho- logical observations on Leber's optic atrophy. Brain 1966;89:15-26.

12 Wilson J. Leber's hereditary optic atrophy-some clinical and aetiological considerations. Brain 1963;86:347-62.

13 Miller DH, Ormerod IEC, McDonald W, et al. The early risk of multiple sclerosis after optic neuritis. J Neurol Neurosurg Psychiatry 1988;51:1569-71.

14 Miller DH, Newton MR, van der Poel JC, et al. Magnetic resonance imaging of the optic nerve in optic neuritis. Neurology 1988;38:175-9.

15 Jacobs L, Kinkel PR, Kinkel WR. Silent brain lesions in patients with isolated optic neuritis. Arch Neurol 1986;43:452-5.

16 Ormerod IEC, Miller DH, McDonald WI, et al. The role of NMR imaging in the assessment of multiple sclerosis and isolated neurological lesions. Brain 1987;110:1579-616.

17 Ormerod IEC, McDonald WI, du Boulay EPGH, et al. Disseminated lesions at presentation in patients with optic neuritis J Neurol Neurosurg Psychiatry 1986;49:124-7.

18 Holt IJ, Miller DH, Harding AE. Restriction endonuclease analysis of leukocyte mitochondrial DNA in Leber's optic atrophy. J Neurol Neurosurg Psychiatry 1988;51:1075-7.

19 McDonald WI. The pathophysiology of multiple sclerosis. In McDonald WI, Silberberg DH, eds. Multiple Sclerosis. Guildford: Butterworths, 1986:112-33.

20 Barnes D, McDonald WI, Johnson G, Tofts PS, Landon DN Quantitative nuclear magnetic resonance imaging; characterisation of experimental cerebral oedema. J Neurol Neurosurg Psychiatry 1987;50:125-33.

21 Barnes D, McDonald WI, Landon DN, Johnson G. Characterisation of experimental gliosis by quantitative nuclear magnetic resonance imaging. Brain 1988;111:83-94. 